\title{
Lung cancer: a potential role for dentists
}

\author{
E. Anne Field ${ }^{* 1}$ and John K. Field ${ }^{2}$
}

\section{Key points}

Lung cancer is the leading cause of death from cancer worldwide, with a five-year survival rate of approximately $15 \%$ in the UK.
Lung cancer has failed to attract the attention and resources given to other cancers and has been stigmatised by a culture of blame because of its association with smoking.
Dentists and their teams could potentially play a role in the public perception, prevention and detection of lung cancer.

\begin{abstract}
Lung cancer is the leading cause of death from cancer worldwide and causes more deaths than breast, colorectal and cervical cancers combined. The five-year survival rate for lung cancer in the UK is approximately $15 \%$. Over many years, lung cancer has failed to attract the attention and resources given to other cancers; it has also been stigmatised by a culture of blame, attributable to the sufferer because of smoking. Anyone can develop lung cancer, and between $15 \%$ and $20 \%$ of people with this diagnosis have never smoked. Many patients with lung cancer are diagnosed at late stages; therefore, early detection is pivotal to improving their survival. Dentists and their teams could potentially play a role in the public perception, prevention and detection of lung cancer.
\end{abstract}

Lung cancer is one of the 'Cinderella' cancers that do not get the attention they deserve. This is despite the fact that it is the leading cause of death from cancer worldwide and causes more deaths than breast, colorectal and cervical cancers combined. ${ }^{1}$ The five-year survival rate for lung cancer in Britain is approximately $15 \%$, which lags behind that of other Western nations; currently, there are 35,349 deaths per year from this disease in the UK. ${ }^{2}$ All too often, lung cancer patients are diagnosed at late stages, when their chances of survival are low. Over many years, lung cancer has failed to attract the attention and resources given to other cancers, such as breast and prostate. Asa Murphy, star of the current hit show 'Buddy Holly Lives', has helped raise awareness of lung cancer and eloquently stated: 'There's no Premier League of cancers, where one is more deserving of attention than another.' Lung cancer has also been stigmatised

${ }^{1}$ Honorary Professor, Consultant in Oral Medicine, Liverpool University Dental Hospital, Pembroke Place, Liverpool, L3 5PS, UK; ${ }^{2}$ Clinical Professor in Molecular Oncology, Department of Molecular and Clinical Cancer Medicine, University of Liverpool, The William Duncan Building, 6 West Derby Street, Liverpool, L7 8TX, UK.

${ }^{*}$ Correspondence to: E. Anne Field

Email address: anne.field@rlbuht.nhs.uk

Accepted 14 February 2020

https://doi.org/10.1038/s41415-020-1418-9 by a culture of blame, attributable to the sufferer who, by smoking, has brought this disease upon themselves. When told about a friend or colleague who has a diagnosis of lung cancer, the first response is often: 'did they smoke?' Smoking is, however, strongly associated with many other cancers, including oral cancer, and contributes to the high morbidity and mortality associated with strokes, coronary artery disease and other lung diseases, such as chronic obstructive pulmonary disease (COPD). Although smoking and exposure to tobacco products has long been acknowledged as the major cause of lung cancer, between 15\% and $20 \%$ of sufferers have never smoked. As one young woman diagnosed with incurable lung cancer said, 'you never think you're going to be a young, female non-smoker and get lung cancer.'3

Although there has been a marked decline in smoking in Britain, ${ }^{4}$ due to the success of public health measures, such as raising tax on tobacco products, banning indoor smoking and support for those wanting to quit, there is evidence that smoking initiation in youths remains substantial. ${ }^{5}$ There will also be a large cohort of ex-smokers who will remain at risk from lung cancer for many years.

There are, however, signs that the tide is turning, and lung cancer is finally attracting the attention it deserves. In a recent editorial,
The Times noted that early detection is pivotal to improving lung cancer survival in Britain and that the key to progress lies in screening. ${ }^{6}$

The results of a recent NELSON, large DutchBelgium lung cancer screening trial have shown the efficacy of low-dose CT screening for lung cancer. ${ }^{7}$ The challenge now is to identify the target population in which screening will be acceptable and cost-effective. ${ }^{8}$ Drugs to treat more advanced lung cancer, based on immunotherapy, are being trialled and yielding some promising results, in respect of increasing survival time. ${ }^{9}$

There are four ways that dentists and their teams could potentially play a role in the public perception, prevention and diagnosis of lung cancer. They can firstly increase awareness and challenge misconceptions and stigma about this disease. The message is clear: anyone can develop lung cancer.

Their second potential role is with respect to tobacco cessation and discouraging young people from taking up smoking. Dental teams are already supporting and encouraging dental patients to quit smoking, and training is available for them to offer 'Very Brief Advice' (VBA). ${ }^{10}$

Thirdly, in those geographic areas that are currently being offered targeted screening, as part of healthy lung checks dentists could encourage their patients to participate and accept invitations to attend. ${ }^{11}$ 
Finally, dentists could have a potential role in identifying patients who already have lung cancer. There has been a recent suggestion that pharmacists should be educated to identify 'red flags' for certain cancers, including lung cancer, that may indicate the disease and facilitate earlier diagnosis. ${ }^{12}$ Dentists are, however, in an equally good (if not better) position to undertake this role, as they see many of their patients on a regular one-toone basis. When checking and/or updating their patients' medical history, they may pick up on any signs or symptoms, which might prompt a visit to the patient's GP for further investigation. Unfortunately, lung cancer in its early stages is often asymptomatic, but complaints such as a persistent (or changing) cough, hoarseness of the voice, increasing (or unexplained) shortness of breath, dull pain in the chest or shoulder, recurrent chest infections and unexplained weight loss or loss of appetite should be investigated. ${ }^{13}$ However, many of these signs and symptoms are quite non-specific and could indicate a range of both benign and serious medical conditions.
Last year, it was 25 years ago that Roy Castle, in the terminal stages of lung cancer, embarked upon his 'Tour of Hope' around the UK to raise awareness and funding for lung cancer research. As we enter the second decade of this century, there is hope that lung cancer may emerge from the shadows, cast by stigmatisation and misconceptions, and benefit from progress in early detection and advances in treatment on an equal footing with other cancers.

\section{References}

1. Bray F, Ferlay J, Soerjomataram I et al. Global cancer statistics 2018: GLOBOCAN estimates of incidence and mortality worldwide for 36 cancers in 185 countries. $C A$ Cancer J Clin 2018; 68: 394-424.

2. Cancer Research UK. Lung Cancer mortality statistics. Available at https://www.cancerresearchuk.org/healthprofessional/cancer-statistics/statistics-by-cancer-type/ lung-cancer/mortality (accessed February 2019).

3. Avery R. Buddy. Inspire 2019; 30-31. Available online at https://www.roycastle.org/helpandsupport/inspiremagazine/ (accessed February 2020).

4. Office for National Statistics. Adult smoking habits in the UK: 2018. 2019. Available at https://www. ons.gov.uk/peoplepopulationandcommunity/ healthandsocialcare/healthandlifeexpectancies/bulletins/ adultsmokinghabitsingreatbritain/2018 (accessed February 2020).

5. World Health Organisation. Data and statistics. Available at http://www.euro.who.int/en/health-topics/ disease-prevention/tobacco/data-and-statistics (accessed February 2019).

6. The Times. The Times view on cutting lung cancer deaths: Survival Guide. 2020. Available at https://www. thetimes.co.uk/article/the-times-view-on-cutting-lungcancer-deaths-survival-guide-grjg27pdg (accessed January 2020).

7. De Koning $\mathrm{H}$ J, van de Aalst $\mathrm{C} \mathrm{M}$, de Jong $\mathrm{P} A$ et al. Reduced Lung Cancer Mortality with Volume CT Screening in a Randomized Trial. N Eng/ I Med 2020; 382: 503-513.

8. Duffy S W, Field J K. Mortality Reduction with Low-Dose CT Screening for Lung Cancer. N Eng/ J Med 2020; 382: 572-573.

9. Hu Z, Li M, Chen Z et al. Advances in clinical trials of targeted therapy and immunotherapy of lung cancer in 2018. Trans/ Lung Cancer Res 2019; 8: 1091-1106.

10. BDA. Supporting dental patients to quit smoking: Stoptober 2018. 2018. Available at https://bda.org/ news-centre/blog/supporting-dental-patients-to-quitsmoking-stoptober-2018 (accessed February 2020).

11. NHS. Targeted Screening for Lung Cancer with Low Radiation Dose Computed Tomography: Standard Protocol prepared for the Targeted Lung Health Checks Programme. 2019. Available at https://www.england. nhs.uk/wp-content/uploads/2019/02/targeted-lunghealth-checks-standard-protocol-v1.pdf (accessed April 2019).

12. UCL. Cancer researchers call for ambitious survival targets for the 2020s. 2020. Available at https://www. ucl.ac.uk/news/2020/jan/cancer-researchers-callambitious-survival-targets-2020s (accessed February 2020).

13. Roy Castle Lung Cancer Foundation. Lung Cancer signs and symptoms. Available at https://www.roycastle. org/aboutlungcancer/lungcancersignsandsymptoms/ (accessed February 2020). 